\title{
Research of Reverse Supply Chain Coordination under Asymmetric Information
}

\author{
Ruxiu Zhang \\ Department of Management \\ Xiamen University of Technology \\ Xiamen, China
}

\begin{abstract}
To promote recovery activities, this paper discusses a contract coordination problem by use of game theory, in a reverse supply chain under the guidance of government. Specifically, we present a nonlinear revenue function and consider enterprises' different risk preferences. We analyse collecting effort level, the degree of premium and penalty, minimum return rate, total profits and show how these change with the system parameters. Results show joint investment contract is more effective than shared-saving contract, considering information asymmetry and remanufacturer being the Stackelberg leader. Under the situation of information symmetry or using shared-saving contract, if the total system profitability keeps fixed, changes in profitability of two players have no effect on the system performance. But if remanufacturer has a stronger profitability than collector, the system performance would be better in the condition of adopting joint investment contract. The subsidy of the government can promote the development of remanufacturing industry; while effectively reduce the cost of disposing the waste products.
\end{abstract}

Keywords-reverse supply chain; remanufacturing; coordination contract; information asymmetry

\section{INTRODUCTION}

The forward supply chain (FSC) is composed of a series of activities in the process of converting raw materials to finished goods. In contrast, the reverse supply chain (RSC) refers to the series of activities necessary to retrieve a product from a customer and either dispose of it or recover value [1]. From the perspective of sustainable development, closing the loop between the manufacturer and customers offers the potential to make significant gains in environmental performance. Among the recovery options, remanufacturing can be defined as the process of restoring the quality level of a used product to that of a new product [2]. Goods that are currently remanufactured include computers, copiers, telecommunication equipment, automobile parts, or office furniture. In this paper, we consider remanufactured reverse supply chain which is the process begins from collecting of disposed products and ends with the sale of remanufactured products.

Companies can face many challenges as managing the remanufactured reverse supply chain, such as the uncertainties associated with product returns [3]. The quantity, quality, and timing of return flows couldn't be controlled completely by acquisition prices, so the remanufacturer need consider

The research work was supported by class-A Project of Education Department of Fujian Province of China, under Grant No. JAS150433 and the National Natural Science Foundation of China under Grant No. 7097111. cooperating with the collector to develop the quantity of returned products for gaining the revenue of scaling economics. Therefore, the remanufacturer has the strong will to expand the collecting network. Different from the capacity enlarging of normal supplier, the resource used by expanding or optimizing the collecting network is more special and the final investing revenue could not be expected. Depart from the high risk of investing the collection, the collector often found that the unit collecting cost would increase with rise in quantity of collected products. How to excite the risk-averse collector to increase the quantity of returned products has been a big problem for the risk-neutral remanufacturer.

A growing literature in reverse supply chain, askes how redesigning contracts for remanufacturing products by Game theory can lead to improved performance. Guide and Van Wassenhove think the acquisition of used products is very important for the system management [4]. Reference [5] emphasis the problem of selecting the appropriate reverse channel structure and give the simple coordination mechanisms to match the relation between the collection effort of the retailer and the system profits. Wang and Da design premium and penalty mechanisms to lead the collector to increase its collection quantity [6]. Then they find that there is no effect for the manufacturer offering the collector premium and penalty mechanism. $\mathrm{Xu}$ and $\mathrm{Wu}$ analyses the pricing and product remanufacturing rate decisions in a reverse supply chain [7]. $\mathrm{Hu}$ and Wang discuss the double moral hazard problem in a reverse supply chain and design a linear contract to coordinate the manufacturer and retailer's effort level. Most of related research show there is a linear relation between the effort level and the profit of the system and can't consider fully the different risk attitude of companies in the reverse supply chain [8].

Remanufacturing activities can create economic benefits, environmental benefits and social benefits, with characteristics of positive externalities and public welfare. Therefore, the premium and penalty of the government have a great impact on the collecting and remanufacturing of end-of-life products. [9] The impact of WEEE on the closed-loop supply chain. Mitra and Webster research the effects of the subsidy policy of remanufacturing on manufacturer and remanufacturer [10].

The key contribution of this paper lies in describing the effort level exerted by the collector and its effect to the system as the nonlinear function relation which is closer to the reality situation, considering the risk-averse cost of the collector, and 
analyzing how the contract type and subsidy of government can influence the performance of the remanufactured reverse supply chain.

The paper is organized as follows. In the next section, we propose the model that we research in this paper, and some definitions and assumptions are given. Following the basic model and analytical results are presented. Section 4 develops the coordination mechanism that captures two types of contracts found in the literature. In section 5, the computational simulation is made to illustrate the efficiency of the contracts. Finally, we conclude our paper in section 6.

\section{MODEL ASSUMPTIONS AND NOTATION}

A single collector retrieves the used products and resale them to the single remanufacturer which remanufactures the returns. They both decide independently and have infinite capacity, aiming to maximizing the expected profit. Collector can increase the quantity of returns by the technical innovation and keep the collecting cost unchanged.

We use the following notation throughout the paper: ${ }^{Q}$ will denote the quantity of returns and $Q(x, \varepsilon)=a+\lambda x^{\mu}+\varepsilon$, and $X$ is the level of collecting effort, $a$ is initial quantity, $\lambda$ can be looked as the elastic coefficient of collecting effort. $\mu \in(0,1)$ shows the effect of effort to the quantity, $\varepsilon$ is the normal distribution variables of exogenous uncertain factors, $E(\varepsilon)=0, \operatorname{Var}(\varepsilon)=\delta^{2}$.

The government uses the subsidy mechanism to guide recycling and remanufacturing activities and set a lowest standard for the remanufacturing quantity. The minimum quantity of remanufacturing products is $Q_{0}$, and the subsidy coefficient is $t$. The government subsidy $T=t\left(Q-Q_{0}\right)$, $t \geq 0, Q>Q_{0}$. The marginal revenues of remanufacturer and collector are $\rho_{m}$ and $\rho_{c}$ respectively and their total profits are $\Pi_{m}=\rho_{m} Q(x, \varepsilon)+T$ and $\Pi_{c}=\rho_{c} Q(x, \varepsilon)$ respectively. Because remanufacturing activity is economical and supported by the government, the remanufacturer can assume certain risks and be risk-neural. The collecting market is complex and the collecting quantity is affected by many factors, which lead to the uncertain risk for investing the collecting system. Therefore, most collectors show is risk-averse. $r(>0)$ is the absolute risk aversion coefficient and the utility function of collector with the characteristics of constant absolute risk aversion is $E\left[\Pi_{c}\right]=-e^{-r \Pi_{c}}$. Assumed that risk cost of collector is $\operatorname{Var}\left(\Pi_{c}\right) r / 2$, $\operatorname{Var}\left(\Pi_{c}\right)=\operatorname{Var}\left(\rho_{c} Q(x, \varepsilon)\right)=\rho_{c}^{2} \delta^{2} \quad, \quad$ then collector's certainty equivalent income $\pi_{c}=E\left[\Pi_{c}\right]=\rho_{c}\left(a+\lambda x^{\mu}\right)-x-r \rho_{c}^{2} \delta^{2} / 2$. Since the remanufacturer' certainty equivalent income is $\pi_{m}=E\left[\Pi_{m}\right]=\left(\rho_{m}+t\right)\left(a+\lambda x^{\mu}\right)-t Q_{0}$, the total system's certainty equivalent income $\pi_{T}=E\left[\Pi_{T}\right]=$ $\left(\rho_{c}+\rho_{m}+t\right)\left(a+\lambda x^{\mu}\right)-x-r \rho_{c}^{2} \delta^{2} / 2-t Q_{0}$.

\section{REVERSE SUPPLY CHAIN MODELS ON REMANUFACTURING}

We fist introduce the basic model under the symmetrical information conditions which maximizes profits for the total chain. After that we introduce and analyze shared-cost contract and shared-revenue contract to coordinate the companies in asymmetric information situation. We compare the models with respect to the effort level, collecting quantity and the total system profits. The profit functions of two actors are shown to be concave in the decision variables.

\section{A. Model CC}

In the Model CC, two actors form the centrally coordinated system under the symmetrical information. The central planner optimizes

$$
\max \pi_{T}=\left(\rho_{c}+\rho_{m}+t\right)\left(a+\lambda x^{\mu}\right)-x-r \rho_{c}^{2} \delta^{2} / 2-t Q_{0}
$$

The simultaneous solution of the fist-order condition results in $_{X}{ }^{*}=\left(\lambda \mu\left(\rho_{m}+\rho_{c}+t\right)\right)^{\frac{1}{1-\mu}}$ and $\pi_{T}^{*}=a\left(\rho_{c}+\rho_{m}+t\right)-\frac{r \rho_{c}^{2} \delta^{2}}{2}-t Q_{0}$ $+\left(\frac{1}{\mu}-1\right)\left(\lambda \mu\left(\rho_{m}+\rho_{c}+t\right)\right)^{\frac{1}{1-\mu}}$. From this condition follows Theorem 1.

\section{Theorem 1.}

As $\lambda \mu\left(\rho_{m}+\rho_{c}+t\right)>1$, the optimal effort level would increase with rise in the separate marginal revenue of collector and remanufacturer, the elastic coefficient of collecting effort, the influence degree of recovery effort on the amount of returns, and the optimal total profit has a similar situation. But it also would decrease with the growth of risk aversion coefficient and uncertain factor variance, and the effect of marginal revenue of collector decides by the value of $\Delta=a+k /\left(\rho_{m}+\rho_{c}+t\right) \mu$ $-r \delta^{2} \rho_{c}$. The effect of subsidy is up to the value of $\Delta^{\prime}=a+k /\left(\rho_{m}+\rho_{c}+t\right) \mu-Q_{0}$.

Proof. Let $k=\left(\lambda \mu\left(\rho_{m}+\rho_{c}+t\right)\right)^{\frac{1}{1-\mu}}, \lambda \mu\left(\rho_{m}+\rho_{c}+t\right)>1$, then get the first-order optimality conditions of $X^{*}$ and $\pi_{T}^{*}$ for

$$
\begin{aligned}
\rho_{m}, \lambda, \mu & . \\
\frac{\partial x^{*}}{\partial \rho_{m}} & =\frac{\partial x^{*}}{\partial \rho_{c}}=\frac{\partial x^{*}}{\partial t}=\frac{k}{(1-\mu)\left(\rho_{m}+\rho_{c}+t\right)}>0 \quad, \quad \frac{\partial x^{*}}{\partial \lambda}=\frac{k}{(1-\mu) \lambda}>0, \\
\frac{\partial x^{*}}{\partial \mu} & =\frac{\left(1-\mu+\mu \ln \left(\lambda \mu\left(\rho_{m}+\rho_{c}\right)\right)\right) k}{(1-\mu)^{2} \mu}>0 \quad \frac{\partial \pi_{T}^{*}}{\partial \lambda}=\frac{k}{\lambda \mu}>0 \\
\frac{\partial \pi_{T}^{*}}{\partial \mu} & =\lambda\left(\rho_{m}+\rho_{c}\right) k^{\mu} \ln k>0 \quad \frac{\partial \pi_{T}^{*}}{\partial \rho_{m}}=a+\frac{k}{\left(\rho_{m}+\rho_{c}+t\right) \mu}>0 \\
\frac{\partial \pi_{T}^{*}}{\partial \rho_{c}} & =a+\frac{k}{\left(\rho_{m}+\rho_{c}+t\right) \mu}-r \delta^{2} \rho_{c} \frac{\partial \pi_{T}^{*}}{\partial t}=a+\frac{k}{\left(\rho_{m}+\rho_{c}+t\right) \mu}-Q_{0},
\end{aligned}
$$

\section{B. Model CS}

Under the situation of information asymmetric, two actors decide independently and have different maximizing aims. We suppose the remanufacturer is the Stackelberg leader and can choose two kinds of contracts to coordinate the system. After that, the collector decides its optimal effort level. 
In this model, we consider cost-sharing contract which the ratio of remanufacturer sharing the cost of collecting effort is $\phi$.The collector pays a fraction $1-\phi$ of the cost incurred by expanding or improving the collecting network. The collector optimizes

$$
\max \pi_{c}=\rho_{c}\left(a+\lambda x^{\mu}\right)-(1-\varphi) x-r \rho_{c}^{2} \delta^{2} / 2
$$

The simultaneous solution of the fist-order condition results in $x^{*}=\left(\frac{\lambda \mu \rho_{c}}{1-\phi}\right)^{\frac{1}{1-\mu}}$. The remanufacturer's problem can be stated

as

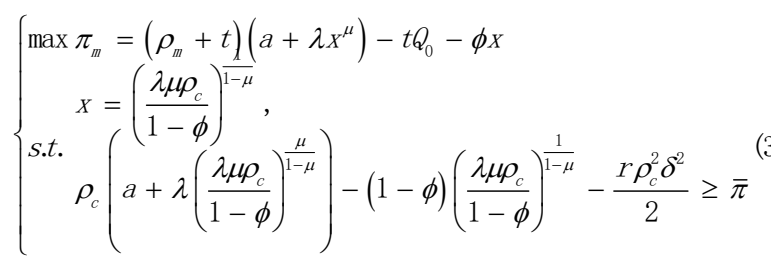

$\bar{\pi}$ is the retainable profit of collector which makes sure of its attending this system. Again, the remanufacturer's first-order conditions characterize the unique best response. If $\frac{\rho_{m}+t-(1-\mu) \rho_{c}}{\rho_{m}+t+\mu \rho_{c}} \geq 1-\left(\frac{(1-\mu)\left(\lambda \mu \rho_{c}\right)^{\frac{1}{1-\mu}}}{a \rho_{c}-\bar{\pi}-r \rho_{c}^{2} \delta^{2} / 2}\right)^{\frac{1-\mu}{\mu}} \quad, \quad \phi^{*}=$ $\frac{\rho_{m}+t-(1-\mu) \rho_{c}}{\rho_{m}+t+\mu \rho_{c}}, x^{*}=\left(\lambda \mu\left(\mu \rho_{c}+\rho_{m}+t\right)\right)^{\frac{1}{1-\mu}}$. Further, we can $\quad$ get $\quad \pi_{c}^{*}=a \rho_{c}+\left(\frac{(1-\mu) \rho_{c}}{\mu\left(\rho_{m}+t+\mu \rho_{c}\right)}\right)\left(\lambda \mu\left(\mu \rho_{c}+\rho_{m}+t\right)\right)^{\frac{1}{1-\mu}}$ $-\frac{r \rho_{c}^{2} \delta^{2}}{2}, \pi_{m}^{*}=a\left(\rho_{m}+t\right)+\frac{(1-\mu)}{\mu}\left(\lambda \mu\left(\mu \rho_{c}+\rho_{m}+t\right)\right)^{\frac{1}{1-\mu}}-t Q_{0}$ $\pi_{\tau}^{*}=a\left(\rho_{m}+\rho_{c}+t\right)+\frac{(1-\mu)\left[(1+\mu) \rho_{c}+\rho_{m}+t\right]}{\mu\left(\mu \rho_{c}+\rho_{m}+t\right)}\left(\lambda \mu\left(\mu \rho_{c}+\rho_{m}+t\right)\right)^{\frac{1}{1-\mu}}$ $-\frac{r \rho_{c}^{2} \delta^{2}}{2}-t Q_{0}$.

$$
\begin{gathered}
\text { If } \quad \frac{\rho_{m}+t-(1-\mu) \rho_{c}}{\rho_{m}+t+\mu \rho_{c}} \\
<1-\left(\frac{(1-\mu)\left(\lambda \mu \rho_{c}\right)^{\frac{1}{1-\mu}}}{a \rho_{c}-\bar{\pi}-r \rho_{c}^{2} \delta^{2} / 2}\right)^{\frac{1-\mu}{\mu}} \\
\phi^{*}=1-\left(\frac{(1-\mu)\left(\lambda \mu \rho_{c}\right)^{\frac{1}{1-\mu}}}{a \rho_{c}-\bar{\pi}-r \rho_{c}^{2} \delta^{2} / 2}\right)^{\frac{1-\mu}{\mu}}, x^{*}=\left(\frac{\left(a \rho_{c}-\bar{\pi}-r \rho_{c}^{2} \delta^{2} / 2\right)^{1-\mu}}{\lambda \mu \rho_{c}(1-\mu)}\right)^{\frac{1}{\mu}} .
\end{gathered}
$$

From this condition follows Theorem 2.

Theorem 2.

$$
\operatorname{As} \frac{\rho_{m}+t-(1-\mu) \rho_{c}}{\rho_{m}+t+\mu \rho_{c}} \geq 1-\left(\frac{(1-\mu)\left(\lambda \mu \rho_{c}\right)^{\frac{1}{1-\mu}}}{a \rho_{c}-\bar{\pi}-r \rho_{c}^{2} \delta^{2} / 2}\right)^{\frac{1-\mu}{\mu}} \text {, the optimal }
$$

sharing ratio would increase with rise in the marginal revenue of remanufacturer, the influence degree of recovery effort on the amount of returns, and decline with rise in the marginal revenue of collector. The optimal effort level of Model CS is lower than that of Model CC and increases with the elastic coefficient of collecting effort, the actor's separate marginal revenues.
Proof. Let get the first-order optimality conditions of $\chi^{*}$ for $\rho_{m}, \rho_{c}, t, \mu, \lambda$, and $\phi^{*}$ for $\rho_{m}, \rho_{c}, \mu$.

$$
\text { As } \quad \mu \in(0,1) \quad, \quad \mu \rho_{c}<\rho_{c} \quad, \quad\left(\lambda \mu\left(\mu \rho_{c}+\rho_{m}\right)\right)^{\frac{1}{1-\mu}}
$$

$<\left(\lambda \mu\left(\rho_{c}+\rho_{m}\right)\right)^{\frac{1}{1-\mu}}$, the cost-sharing contract cannot realize the system coordinate completely. $\frac{\partial \phi^{*}}{\partial \rho_{m}}=\frac{\partial \phi^{*}}{\partial t}=\frac{\rho_{c}}{\left(\rho_{m}+t+\mu \rho_{c}\right)^{2}}>0, \frac{\partial \phi^{*}}{\partial \rho_{c}}=\frac{-\left(\rho_{m}+t\right)}{\left(\rho_{m}+t+\mu \rho_{c}\right)^{2}}<0$, $\frac{\partial \phi^{*}}{\partial \mu}=\frac{\rho_{c}^{2}}{\left(\rho_{m}+t+\mu \rho_{c}\right)^{2}}>0$

$\frac{\partial x^{*}}{\partial \rho_{m}}=\frac{\partial x^{*}}{\partial t}=\frac{k}{(1-\mu)\left(\rho_{m}+t+\mu \rho_{c}\right)}>0 \quad, \quad \frac{\partial x^{*}}{\partial \rho_{c}}=$ $\frac{\mu k}{(1-\mu)\left(\rho_{m}+t+\mu \rho_{c}\right)}>0 \quad, \quad \frac{\partial x^{*}}{\partial \lambda}=\frac{k}{(1-\mu) \lambda}>0 \quad$, $\frac{\partial x^{*}}{\partial \mu}=\frac{\left(1-\mu+\mu \ln \left(\lambda \mu\left(\rho_{m}+t+\mu \rho_{c}\right)\right)\right) k}{(1-\mu)^{2} \mu}>0$.

Theorem 3.

$$
\text { As } \frac{\rho_{m}+t-(1-\mu) \rho_{c}}{\rho_{m}+t+\mu \rho_{c}}<1-\left(\frac{(1-\mu)\left(\lambda \mu \rho_{c}\right)^{\frac{1}{1-\mu}}}{a \rho_{c}-\bar{\pi}-r \rho_{c}^{2} \delta^{2} / 2}\right)^{\frac{1-\mu}{\mu}} \text {, the }
$$
optimal sharing ratio and effort level would decline with rise in risk aversion coefficient and uncertain factor variance, and the elastic coefficient of collecting effort.

\section{Model RS}

In this model, two actors share the revenue from the joint venture. The remanufacturer pays a fixed fee $M$ independent of the collecting effort and remain fraction $1-\beta$ of the total revenues. The collector accepts a fraction $\beta$ and then choose its optimal effort level, according the uncertain revenue. The collector's problem can be stated as

$$
\begin{gathered}
\max \pi_{c}=M+\beta\left[\left(\rho_{c}+\rho_{m}+t\right)\left(a+\lambda x^{\mu}+\varepsilon\right)-t Q_{0}\right]-x- \\
\frac{r \beta^{2}\left(\rho_{c}+\rho_{m}+t\right)^{2} \delta^{2}}{2}
\end{gathered}
$$

The simultaneous solution of the fist-order condition results in $x^{*}=\left(\beta \lambda \mu\left(\rho_{c}+\rho_{m}+t\right)\right)^{\frac{1}{1-\mu}}$.Then the remanufacturer's problem is

$\left\{\begin{array}{l}\max \pi_{m}=(1-\beta)\left(\rho_{c}+\rho_{m}+t\right)\left(a+\lambda x^{\mu}\right)-t Q_{0}-M \\ \text { s.t. } x=\left(\alpha \lambda \mu\left(\rho_{c}+\rho_{m}+t\right)\right)^{\frac{1}{1-\mu}}, \\ M+\beta\left(\rho_{c}+\rho_{m}+t\right)\left(\begin{array}{l}a+\lambda x^{\mu} \\ a\end{array}\right)-x-r \beta^{2}\left(\rho_{c}+\rho_{m}+t\right)^{2} \delta^{2} / 2 \geq \bar{\pi}\end{array}\right.$

$\bar{\pi}$ is the retained earnings of collector. Under the optimal condition, the participation restriction of collector is established. $\quad M^{*}=\bar{\pi}-\left(\beta\left(\rho_{c}+\rho_{m}+t\right)\left(a+\lambda x^{\mu}\right)-x-\right.$ $\left.r \beta^{2}\left(\rho_{c}+\rho_{m}\right)^{2} \delta^{2} / 2\right)+t Q_{0}$.We can get that $\beta^{*}$ satisfy 
$\beta^{\frac{3 \mu-2}{1-\mu}}-\beta^{\frac{2 \mu-1}{1-\mu}}=r(1-\mu) \sigma^{2}\left(\rho_{c}+\rho_{m}+t\right)^{2}\left(\lambda \mu\left(\rho_{c}+\rho_{m}+t\right)\right)^{\frac{1}{\mu-1}}$

$=r(1-\mu) \sigma^{2}\left(\rho_{c}+\rho_{m}\right)^{2}\left(\lambda \mu\left(\rho_{c}+\rho_{m}\right)\right) \frac{1}{\mu-1} \quad$.If $\quad \mu=0.5 \quad, \quad \beta^{*}$

$=\lambda^{2} /\left(\lambda^{2}+2 r \delta^{2}\right), x^{*}=\lambda^{6}\left(\rho_{c}+\rho_{m}+t\right)^{2} / 4\left(\lambda^{2}+2 r \delta^{2}\right)^{2}$.

\section{COMPUTATIONAL EXAMPLES AND ANALYSIS}

In order to compare the coordinate effect of two contracts and further test the above results, we construct an example which the specific parameters are set as follows: $\lambda=4, \mu=0.5, \delta=10, \mathrm{r}=0.5, \bar{\pi}=3500$, $a=500, Q_{0}=400$. We get following conclusions according the data analysis, showing from Fig.1.

Proposition 1. In the model CC, the system performance would be better if $\rho_{m}>\rho_{c}$ and first increase and then decline with $\rho_{c}$ because of risk cost. And when $\rho_{m}<\rho_{c}$, only the effect of collecting effort level on the system profit reach a certain degree, can the collector have motive to invest the collecting network.

Proposition 2.The coordinated effect of Model CS is better than that of Model RS if $\rho_{m}>\rho_{c}$ and if the sum of $\rho_{m}$ and $\rho_{c}$ keeps same, the fraction of cost-sharing and effort level would both increase with rise in the difference between $\rho_{m}$ and $\rho_{c}$. As the collector belongs of the type of risk-averse, joint investment contract would lead to reduced risk.

Proposition3.The profits of the system and the two enterprisers will increase with $t, \rho_{m}$ and $\rho_{c}$ under Model CS, and only the remanufacturer's profits will increase with $t, \rho_{m}$ and $\rho_{c}$ under Model RS.

Proposition 4. If $\rho_{m}=\rho_{c}$, Model RS is the best, because the risk cost would increase with $\rho_{c}$ when the sum of $\rho_{m}$ and $\rho_{c}$ is fixed.

Proposition 5. The subsidy of the government can promote the collecting and remanufacturing activities. $x^{*}$ will increase with $t$.

(a) The change of $\pi_{T}^{*}$ with $\lambda$

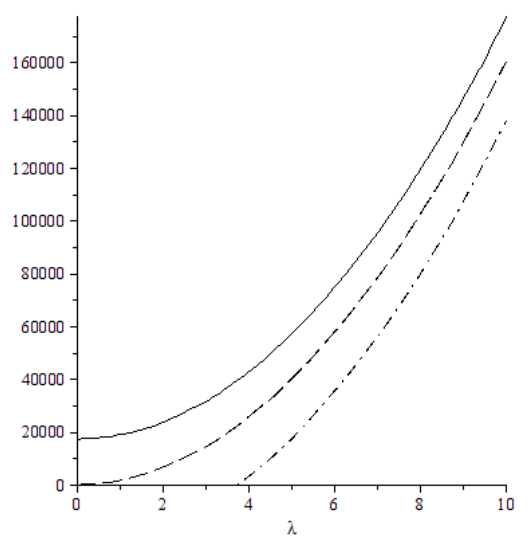

(b) The change of $\pi_{T}^{*}$ with $\mu$

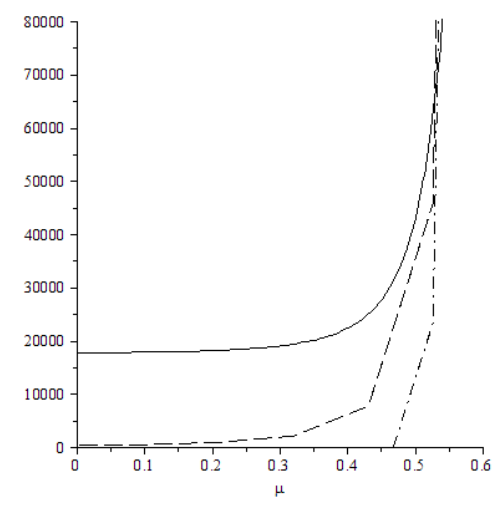

(c) The change of $\pi_{T}^{*}$ with $\rho_{c}$

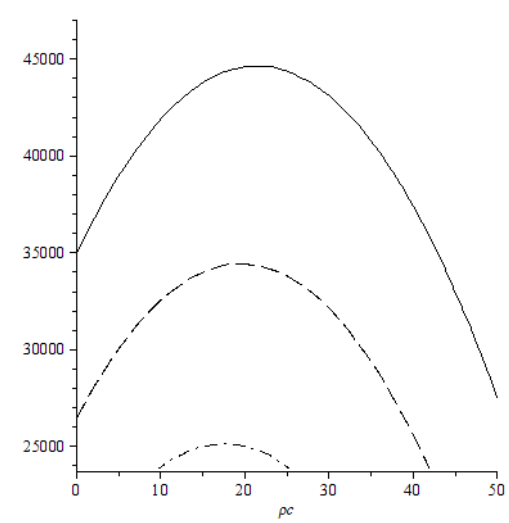

Fig.1. The effect of $\rho_{m}, \rho_{c}, \mu, \lambda$ on $\pi_{T}^{*}$ in Model CC

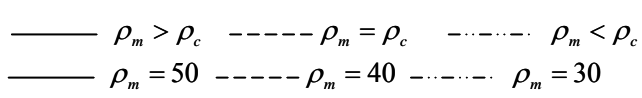

\section{CONCLUSIONS}

This paper discusses a contract coordination problem of remanufacturing reverse supply chain considering different risk preference of players. We analyse collecting effort level, total profits and show how these change with the system parameters. Joint investment contract is more effective than shared-saving 
contract in promoting the collector to work hard for more returned products. And if remanufacturer has a stronger profitability than collector, the system performance would be better. To realize sustainable development, government should develop the consumer's evaluation to remanufacturing product and give more support to remanufacturing activity than collecting.

\section{ACKNOWLEDGMENT}

The research work was supported by class-A Project of Education Department of Fujian Province of China, under Grant No. JAS150433 and the National Natural Science Foundation of China under Grant No. 7097111.

\section{REFERENCES}

[1] V.D.R. Guide and L. N. Wassenhove, "The reverse supply chain", Harv. Bus. Rev. vol. 80, pp.25-26, February 2002.

[2] M.E.Ferguson and L. B.Toktay, "The Effect of Competition on Recovery Strategies”, Prod. Oper. Manag., vol.15, pp.351-368, September 2006.
[3] A.Atasu and M. Sarvary and L. N. Wassenhove, "Remanufacturing as a marketing strategy”. Manag. Sci., vol.54, pp.1731-1746, October 2008.

[4] V. D. R.Guide and L. N.Wassenhove, "Managing product returns for remanufacturing”, Prod. Oper. Manag., vol.10, pp.142-155, June 2001.

[5] R. C.Savaskan and S.Bhattacharya and L. N. Wassenhove, "Closed-loop supply chain models with product remanufacturing”. Manag. Sci., vol.50, No.2, pp. 239-252, February 2004.

[6] W. B.Wang and Q. L.Da, "The comparison of premium, penalty and premium and penalty mechanism for Remanufacturing reverse supply chain coordination". J. Ind. Eng. Eng. Manag., vol.24, pp.48-52, February 2010.

[7] B.Xu and M.Wu, "Decision Models of Product Remanufacturing, Pricing and Analysis of Supply Chain Coordination". Oper. Res. Manag. Sci., vol.20, pp.57-63, April 2011.

[8] X. P. Hu and Y. G.Wang, "Incentive recycling contracts with double moral hazard in reverse supply chain", Ind. Eng. J., vol.15, pp.24-29, June 2012.

[9] Hammond D, Beullens P. Closed-loop supply chain network equilibrium under legislation[J]. European Journal of Operational Research, 2007, 183(2):895-908.

[10] Mitra S, Webster S. Competition in remanufacturing and the effects of government subsidies[J]. International Journal of Production Economics, 2008, 111(2):287-298 